\title{
Disseminated Discoid Lupus Erythematosus and Hypothyroidism in a Vitiligo Vulgaris Patient: A Rare Coexistence
}

\author{
Paudel $\mathbf{S}^{1}$, Neupane $\mathbf{S}^{2}$ \\ Registrar, ${ }^{1}$ Department of Dermatology, ${ }^{2}$ Department of Pathology, Civil Service Hospital, Kathmandu, Nepal.
}

\begin{abstract}
Vitiligo vulgaris is a disease with multiple etiological factors including genetic, autoimmunity and several environmental factors. It has a huge psychological burden in the patient. Discoid lupus erythematosus (DLE) in the other hand can be quite disfiguring as well. Thyroid disease along with these chronic skin diseases if occurs in a same patient is really disappointing to him and his family. We report a case of 55years old man with such a rare coexistence in a patient.
\end{abstract}

Key words: Autoimmunity; Lupus Ertythematosus; Discoid; Nepal; Thyroid Diseases; Vitiligo

\section{Introduction}

$\mathrm{V}$ itiligo is an acquired disease with multiple etiological factors most compelling evidence being autoimmune basis. ${ }^{1}$ Lupus Erythematosus consists of diverse illnesses with the development of autoimmunity directed at the molecular constituents of nucleosomes and ribonucleoproteins. ${ }^{2}$ Vitiligo is found to be associated with several autoimmune diseases. ${ }^{3}$ The coexistence of vitiligo andDiscoid lupus erythematosus (DLE)has been infrequently reported and the third autoimmune entity in the same patient is rarely reported. ${ }^{4}$ This is an important consideration as the presence of one disorder may affect the treatment and prognosis of the other.

\section{Case Report}

A man of 55 years with stable vitiligo vulgaris (BSA $5 \%$ approx) of 15 years involving scalp, both hands, forearms, trunk, both legs and both feet;presented with us with gradual onset of itchy scaly raised skin lesions, few over the old vitiligo macules and mostly at the new sites over face, scalp, ears and chest in the last 2 months period.

Financial disclosure: None.

Conflict of interest to disclosure: None declared.

Address of Correspondence

Dr. Sushil Paudel M.D

Registrar, Department of Dermatology

Civil Service Hospital, Kathmandu

E-mail: paudelsushil@gmail.com
On examination he was found to have multiple well defined scaly erythematous plaques of variable sizes (ranging from $1 \times 1 \mathrm{~cm}$ to $7 \times 10 \mathrm{~cm}$ ) over the face, mostly periauricular areas, lips, scalp and the upper chest (Figures 1 and 2). He also had multiple well defined depigmented macules of varying sizes over hands, forearms, fingers, face, scalp, trunk and lower limbs. Oral cavity had ill defined patches over the palate which he had not observed. Hair and nails were normal. His systemic examination was normal.

Clinical diagnosis of disseminated discoid lupus erythematosus (DDLE) with vitiligo was made and asked for few investigations and a skin biopsy was planned. His TSH was elevated and FreeT4 was depressed. All other investigations including ANA came to be normal. Skin biopsy in H\&E stain revealed the features of DLE (Figures 3 and 4).

Submitted: $3^{\text {rd }}$ October 2019

Accepted: $30^{\text {th }}$ November 2019

Published: $7^{\text {th }}$ October 2020

How to cite this article

Paudel S, Neupane S. Disseminated discoid lupus erythematosus and hypothyroidism in a vitiligo vulgaris patient: A rare coexistence. Nepal Journal of Dermatology, Venereology and Leprology 2020;18(1):56-9. https://doi. org/10.3126/njdvl.v18i1.30317.

\section{(c) (i)}

Licensed under CC BY 4.0 International License which permits use, distribution and reproduction in any medium, provided the original work is properly cited. 
He was started on topical steroid of midpotent strength along with antihistamines and sunscreen and was asked to avoid sun. Thyroid hormone replacement was done after a physician consultation. But he returned in just five days with thecomplaint of unrelenting itching. Then he was started on oral prednisolone starting from $0.5 \mathrm{mg} / \mathrm{kg}$ dose for two weeks and tapered in next two weeks along with hydroxychloroquin 200mg per day. Topical tacrolimus $0.1 \%$ was started for both DLE and vitiligo lesions.

His follow up in two and four months (Figures 5 and 6) showed good response in DLE lesions and vitiligo of face. His vitiligo of hands and scalp was static. His follow up in one year (Figure 7) showed very good resolution of DLE lesions. Thyroid function test is normal on $25 \mathrm{mcg}$ of thyroxin. Now he is put under only Sunscreen and moisturizers.

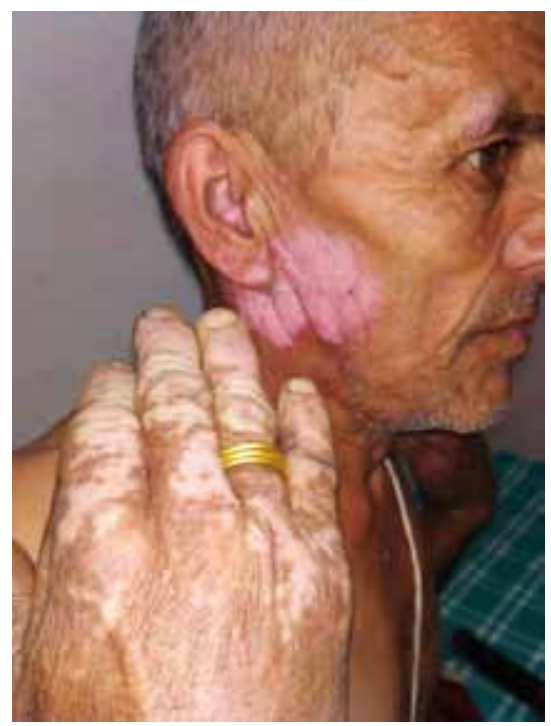

Figure 1: Vitiligo macules over hands and DLE plaque over right cheek, ear and brow.

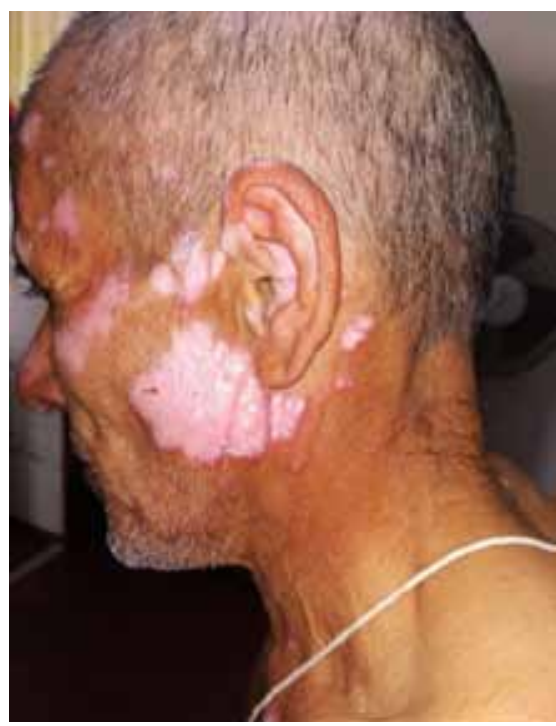

Figure 2: Multiple DLE plaques over left cheek, ear, behind ear and forehead

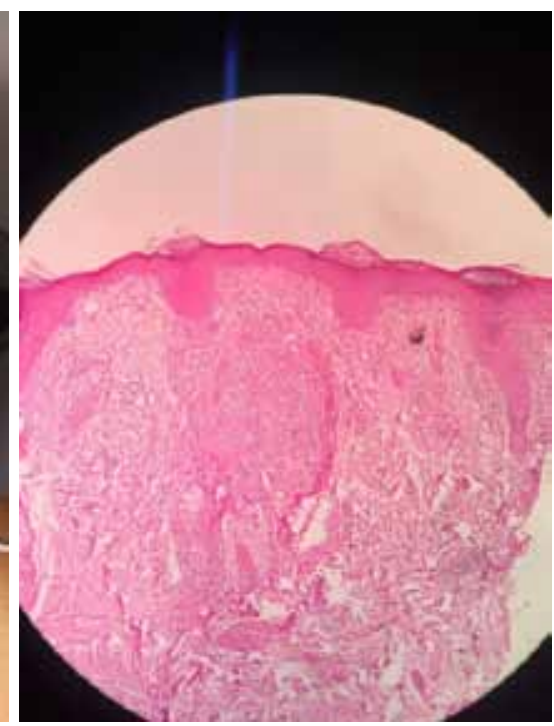

Figure 3: Photomicrograph of $\mathrm{H}$ and $\mathrm{E}$ stained section (10X) shows hyperkeratosis, irregular acanthosis and follicular plugging in the epidermis. Dermis shows perifollicular and perivascular lympho-histiocytic infiltrate.

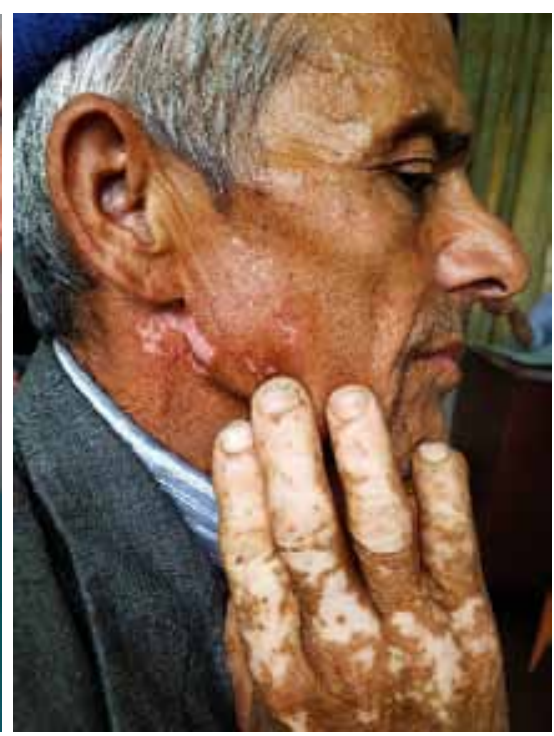

Figure 6: After 4 months of treatment
Figure 5: After 2 months of treatment

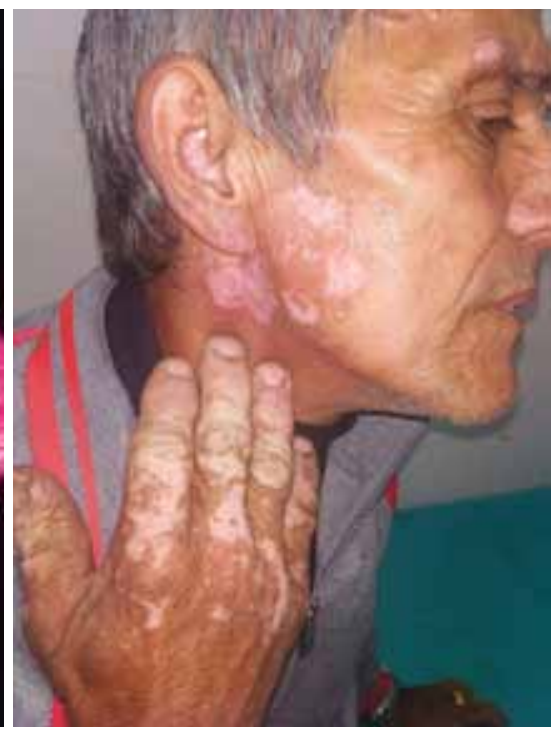

Figure 4: Photomicrograph of $\mathrm{H}$ and $\mathrm{E}$ stained section (40X) shows follicular plugging, basal vacuolation and perifollicular lymphocytic infiltrate 


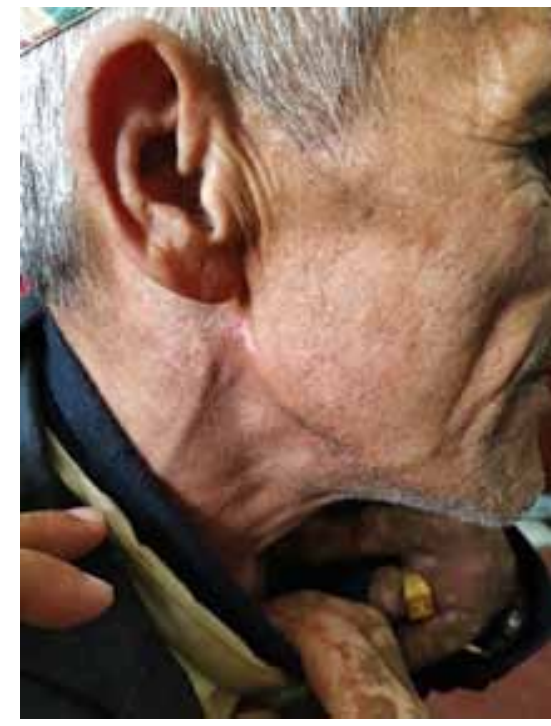

Figure 7: At one year follow up

\section{Discussion}

Several studies have noted the coexisting vitiligo and DLE in various clinical spectrum. ${ }^{3,4,5}$ A few of them have reported multiple similar autoimmune conditions coexisting in one person. Coexisting vitiligo, DLE and autoimmune thyroid disease is very uncommonly reported. ${ }^{4}$

In a cross sectional study among 1098 vitiligo patients, $19.8 \%$ of them were found to have at least one other autoimmune condition, commonest being thyroid disease (12.9\%). Others being alopecia areata, inflammatory bowel diseases, pernicious anemia, SLE, Guillain-Barre syndrome, DLE (0.2\%), linear morphea, myasthenia gravis and Sjögren syndrome. ${ }^{3}$

\section{References}

1. Stanca BA, Richard SA, David NA. Vitiligo. In: Goldsmith LA, Katz SI, Gilchrest BA, Paller AS, Leffel DJ, Wolff $K$, editors. Fitzpatrick's dermatology in general medicine. 8th ed. McGrawHill; 2012.p.792.

2. Melissa IC, Richard DS. Lupus Erythematosus. In: Goldsmith LA, Katz SI, Gilchrest BA, Paller AS, Leffel DJ, Wolff K, editors. Fitzpatrick's Dermatology in General Medicine. 8th ed. McGrawHill;2012.p.1909.

3. Gill L, Zarbo A, Isedeh P, Jacobsen G, Lim HW, Hamzavi I. Comorbid autoimmune diseases in patients with vitiligo: A cross-sectional study.
The reported cases with coexisting vitiligo and DLE in the same patient has been summarized in a table by Devraj et al in $2018 .^{5}$

Newer conditions like multiple sclerosis, Immune Thrombocytopenic Purpura (ITP) and lymphoma have also been associated with vitiligo recently. ${ }^{6}$

Vitiligo and associated autoimmune diseases may have genetic basis. Genetically NALP1 region on chromosome $17 \mathrm{p} 13$ have been thought to contribute environmental factors in occurrence of autoimmune diseases in patients with vitiligo. ${ }^{7}$

A study conducted on 130 patients with multiples skin conditions like psoriasis, lichen planus, DLE, pemphigus, vitiligo and alopecia areata showed statistically significant alteration in serum lgG, IgM and circulating immune complexesdepending on the extent of the lesion and lowered significantly to almost normal values following treatment, thereby confirming the role of immunity in the pathogenesis of these skin disorders. ${ }^{8}$

Topical steroids and calcineurin inhibitors can be used for both the conditions. Oral steroids improve both the conditions but long term treatment may not be suitable. Oral hydroxychloroquin is the mainstay of treatment for DDLE. ${ }^{2}$ Phototherapy is considered as first line treatment for vitiligo but can aggravate the lupus erythematosus. ${ }^{1}$

\section{Conclusion}

Vitiligo is a common condition. When approaching such patients, dermatologists should look for the possibility of other autoimmune conditions as they are not uncommon.

J Am Acad Dermatol 2016 Feb;74(2):295-302. https://doi.org/10.1016/j.jaad.2015.08.063

4. Sharma S, Sarkar R, Garg VK, Bansal S. Coexistence of lip-tip vitiligo and disseminated discoid lupus erythematosus with hypothyroidism: Need for careful therapeutic approach. Indian Dermatol Online J 2013;4(2):112-4. https://doi. org/10.4103/2229-5178.110636

5. Devaraj $Y$, Sathyanarayana BD, Swaroop MR, Sowmya Shree H, Ravindranath M, Ghosh A. Discoid lupus erythematosus in acroorificial vitiligo. Int J Res Dermatol 2018;4:456-9. https://doi.org/10.18203/issn.2455-4529. IntJResDermatol20183178 
6. Hadi A, Wang JF, Uppal P, Penn LA, Elbuluk N. Comorbid diseases of vitiligo: A 10-year crosssectional retrospective study of an urban US population. J Am Acad Dermatol 2020 Mar;82(3):628-33. https://doi.org/10.1016/j. jaad.2019.07.036
7. Jin Y, Mailloux CM, Gowan K, Riccardi SL, LaBerge $G$, Bennett DC et al. NALP1 in vitiligo associated multiple autoimmune disease. N Engl J Med 2007;356:121625. https://doi.org/10.1056/ NEJMoa061592

8. Khan N, Maheshwari V, Trivedi I, Kalam A. Immunopathology of skin lesions. Indian J Dermatol Venereol Leprol 2001;67:234-7. 